\title{
ANALYSE DE LA DEFORESTATION DANS LA PERIPHERIE OUEST DE LA RESERVE DE BIOSPHERE DU DJA AU CAMEROUN, A PARTIR D'UNE SERIE MULTI-ANNUELLE D'IMAGES LANDSAT
}

\author{
Eric W. Tegno Nguekam¹, Salomon C. Nguemhe Fils², Joachim Etouna ${ }^{3}$, Simon Njeudeng Tenku \\ ${ }^{1}$ Station Polyvalente de Recherche de Minko, Institut de Recherche Agricole pour le développement, Meyomessala, \\ Cameroun etegnonguekam@gmail.com \\ ${ }^{2}$ Laboratoire de Traitement d'Images (LTI), Institut de Recherche Géologique et Minière (IRGM), Yaoundé, Cameroun \\ ${ }^{3}$ Institut National de Cartographie (INC), Yaoundé, Cameroun \\ ${ }^{4}$ Station Polyvalente de Recherche de Minko, Institut de Recherche Agricole pour le développement, Meyomessala, \\ Cameroun
}

\begin{abstract}
Résumé
Dans cet article, il est question d'évaluer la déforestation dans la périphérie Ouest de la réserve de Biosphère du Dja à travers les techniques de Télédétection et de Système d'Information Géographique. Pour cela, huit images Landsat de date différentes (2011 à 2018) ont été utilisées pour produire les cartes d'occupation du sol, à travers la méthode de classification supervisée et l'algorithme "maximum likelihood ». Les classes d'occupation de sol retenues pour cette classification sont : forêt dense, forêt dégradée, zone de culture, zone marécageuse, zone d'habitation, sol nu et eau. L'analyse des changements a été faite avec la technique de « change detection ». Les résultats de cette étude ont montré que la déforestation a été importante pendant la période d'étude $(2011$ - 2018). Les surfaces forestières se sont principalement transformées en zone de culture, marécage, forêt dégradée, sol nu. Le taux de déforestation observé est de $6,8 \%$ et dénote une importante baisse du couvert forestier dense. L'étude a montré des tendances de déforestation dans cette périphérie. Elle a permis d'observer que les zones tendancieuses sont concentrées principalement autour de certaines activités anthropiques présentes dans cette zone (la plantation agricole SUDCAM, le barrage de Mekin, les lieux habités).
\end{abstract}

Mots-clés : Déforestation, Changement climatique, forêt, tendances de déforestation, images satellites

\begin{abstract}
This article is about assessing deforestation in the western outskirts of the Dja Biosphere Reserve through Remote Sensing and Geographic Information System techniques. To do this, eight Landsat images of different dates (from 2011 to 2018) were used to produce land cover maps, through the supervised classification method and the "maximum likelihood" algorithm. The land cover classes retained for this classification are : dense forest, degraded forest, cultivated area, swampy area, residential area, bare soil and water. The analysis of the changes was made with the technique of "change detection". The results of this study showed that deforestation was significant during the study period (2011 - 2018). Forest surfaces have mainly been transformed into cultivation areas, swamps, degraded forests, bare soil. The observed deforestation rate is $6.8 \%$ and indicates a significant decrease in dense forest cover. The study showed deforestation trends in this outskirts. It made it possible to observe that the tendentious zones are concentrated mainly around certain human activities present in this zone (the agricultural plantation SUDCAM, the Mekin dam, inhabited places).
\end{abstract}

Keywords: Deforestation, Climate change, forest, deforestation trends, satellite images

\section{Introduction}

La majeure partie de la forêt tropicale restante en Afrique est concentrée dans la zone forestière du bassin du Congo, qui est le deuxième écosystème forestier le plus important au monde après le bassin amazonien (Oke 2009). La déforestation observée dans les zones tropicales a pris une ampleur considérable depuis plusieurs décennies. Même si, au cours de l'histoire, toutes les régions ont été concernées par la problématique de la déforestation, celle-ci reste majoritairement centrée sur les forêts tropicales (Verjans 2016). On observe aussi une importante évolution des impacts puisqu'en plus de leur bois, les forêts sont détruites pour leur sol destiné aux cultures. Si les forêts sur pied paraissent n'avoir aucune valeur économique, leur conversion par les agro-entreprises peut générer des bénéfices vertigineux. L'Afrique est devenue une cible pour certains investisseurs avides de terres à bon marché car si défrichées et cultivées, ces forêts pourraient nourrir la population mondiale galopante (Rautner, Leggett, M., and Davis, F 2013). Aujourd'hui, l'une des causes du changement climatique est attribuée à la perte et à la dégradation des forêts.

Les aires protégées forestières aident à conserver les écosystèmes qui fournissent un habitat, un abri, des aliments, des matières premières, du matériel génétique, une barrière contre les catastrophes naturelles, une source stable de ressources et de nombreux autres biens et services propres à l'écosystème (Mansourian, Belokurov, and Stephenson 2017). Les aires protégées forestières jouent aussi un rôle dans la séquestration du carbone atmosphérique, concourant ainsi à la réduction de l'effet de serre. C'est ce qui a fait dire à Evariste Fongnzossie que: "A l'échelle de la forêt, plus le nombre d'espèces d'arbres est élevé, plus la capacité de l'environnement à stocker le carbone est grande " 
La réserve de biosphère du Dja (RBD) au Cameroun, comme toute aire protégée fait face aux enjeux de conservation et de gestion durable. Plusieurs menaces pèsent actuellement sur la RBD, notamment l'expansion de l'agriculture, l'exploitation forestière, la prospection et l'exploitation minière dans sa zone périphérique (Niang, Hamerlynck, and Hance 2006).

L'agriculture sur brûlis avec le défrichement et la conversion de la forêt en champs pratiqués par les populations riveraines porte atteinte à l'équilibre écologique et aux fonctions éco systémiques et altère les services offerts par la forêt. D'autres menaces sur la RBD sont observables, notamment la construction du barrage de Mékin au Nord-Ouest, la perspective d'un projet d'exploitation minière de nickel-cobalt à l'Est et surtout le développement de la vaste plantation d'hévéa de la société Sud-Cameroun Hévéa (SUDCAM) à quelques centaines de mètres de sa frontière occidentale (Greenpeace 2016) sur un territoire d'environ 45200 ha. La pression causée par la plantation d'hévéa est plus importante car d'après Greenpeace, la société aurait détruit 5930 hectares de forêts depuis 2011 avec $42 \%$ de cette déforestation depuis un an et demi.

Cette situation a poussé l'ONG Greenpeace a lancer une alerte portant sur « L'échec de I'UNESCO à protéger la Réserve du Dja au Cameroun " à cause des nombreuses menaces qui pèsent sur elle (Greenpeace 2016). L'article publié par Greenpeace à cette même période faisait également part d'une potentielle déclassification de cette réserve et d'une résiliation du contrat avec le Cameroun sur cet espace d'ici 2018. Mais même si des sources au ministère en charge des forêts rassurent sur la non déclassification potentielle de la réserve du Dja (Engono M 2017), il est vital de s'attaquer aux problèmes qui pourraient nuire à la préservation de la RBD.

Dans un tel contexte, il est important de proposer des solutions pour faciliter la prise en compte de l'ampleur du problème et de ses conséquences, et de ce fait, permettre la prise de bonnes décisions pour la préservation du potentiel forestier autour et dans la RBD. Cela peut se faire à travers un suivi spatio-temporel de la déforestation dans la zone en danger. Quelle peut être alors l'ampleur de l'avancée de la déforestation dans cette périphérie ouest soumise à une telle pression anthropique?

L'observation spatiale de la terre a cet avantage qu'elle permet d'estimer les dynamiques de certains composants de l'environnement telles que les forêts, les parcelles de culture, etc. En effet, les archives d'images satellites et les images les plus récentes permettent d'avoir une vue globale, régionale ou locale des changements à la surface de la terre. D'où l'implication de la télédétection dans cette étude.

\section{Travaux similaires}

Plusieurs auteurs ont également travaillé sur l'évaluation de la déforestation à travers des techniques de télédétection. C'est l'exemple de

(Solefack et al. 2012) qui utilise également des images satellitaires Landsat prises entre 1978 et 2007 et modélise l'influence des facteurs environnementaux (altitude, pente, densité humaine) pour l'estimation de la déforestation à travers la caractérisation des changements d'occupation du sol sur le mont Oku (Cameroun). Sur le plan méthodologique, les cartes d'occupation du sol ont été produites à travers les images Landsat et combinées aux variables topographiques et humaines pour estimer la déforestation. Les résultats de cette étude montrent une régression continue de près de $62 \%$ sur la période d'étude.

Par ailleurs, (N'Da et al. 2008) propose de caractériser et de cartographier la couverture végétale du Parc de la Marahoué, puis d'évaluer sa dynamique forestière de 1986 à 2003, à partir de l'imagerie spatiale, afin de mieux comprendre les causes de la réduction des forêts. La méthodologie utilisée pour la discrimination des forêts était la classification supervisée des images Landsat par la méthode de maximum de vraisemblance. Cette étude met en évidence l'impact des défrichements agricoles sur la perte des surfaces forestières pendant la période d'étude, tout ceci sous l'influence de la forte croissance démographique.

Enfin, (Ngamba, et al. 2016), a travaillé sur l'analyse prospective de la déforestation estimée par télédétection dans la réserve de biosphère de Yangambi au Congo. L'objectif de son étude était de déterminer la dynamique spatiotemporelle des forest et les tendances jusqu'en 2065 dans la Réserve de Biosphère de Yangambi. La méthodologie employée était basée sur la comparaison de deux cartes d'occupation du sol, produites grâce aux images Landsat $(1986,2015)$ et l'intégration de quelques variables pour l'estimation de la déforestation à I'horizon 2065. Les résultats montrent une régression significative des formations forestières au profit des classes anthropisées et le présage d'une régression alarmante et continuelle si des mesures conséquentes ne sont pas mises en œuvre.

\section{Objectifs}

L'objectif de cette étude est d'évaluer la perte du couvert végétal dans la périphérie Ouest de la RBD entre 2011 et 2018 à l'aide d'une série multi annuelle d'image Landsat. Spécifiquement, il est question de :

Faire une cartographie de l'occupation du sol pour chaque année choisie ;

- Evaluer la déforestation apparue dans la période d'étude ;

Identifier les zones à forte tendance de déforestation.

\section{Matériels et méthodes \\ 4.1. Zone d'étude}

La zone retenue pour cette étude est la périphérie Ouest de la Réserve de Biosphère du Dja, d'une superficie de 297943,16 Ha. Elle a été choisie à cause de la pression intense exercée sur le couvert végétal par les activités anthropiques qui y ont lieu.

Administrativement, elle se trouve dans la région du Sud Cameroun, dans le département du Dja et Lobo et plus précisément dans l'arrondissement de Meyomessala. Cette commune est limitrophe avec les communes d'Endom, Zoétélé, Mayomessi, Djoum, Bengbis et Sangmelima (CVUC 2014) (Figure 1). Sur le plan climatique, La commune de Meyomessala a un climat humide de type équatorial guinéen classique (PNDP 2016). Le relief appartient à celui du plateau sud camerounais avec une altitude moyenne de $600 \mathrm{~m}$. Le réseau hydrographique de la RBD est très influencé par 
le fleuve Dja. Celui-ci constitue une limite naturelle au Nord, à l'Ouest et au Sud de la réserve et forme ainsi la " boucle du Dja " (MINFOF and UICN 2015). Le diagnostic fait par le PNDP dans cette commune indique que la commune est caractérisée par une population d'environ 65869 habitants. La densité de la population humaine de la région du Dja reste faible dans son ensemble. Les agglomérations les plus importantes en dehors de Lomié ( $3 \mathrm{~km}$ de la RBD) et Somalomo (500 $\mathrm{m}$ ), sont assez éloignées de la réserve : Sangmélima 70 $\mathrm{km}$ environ, Meyomessala $20 \mathrm{~km}$, Djoum $30 \mathrm{Km}$, Bengbis $15 \mathrm{~km}$. Cependant, de nombreux villages entourent la réserve et y exercent une pression plus ou moins importante sur le plan agricole, de la chasse et de la collecte d'autres produits forestiers non ligneux (MINFOF and UICN 2015).

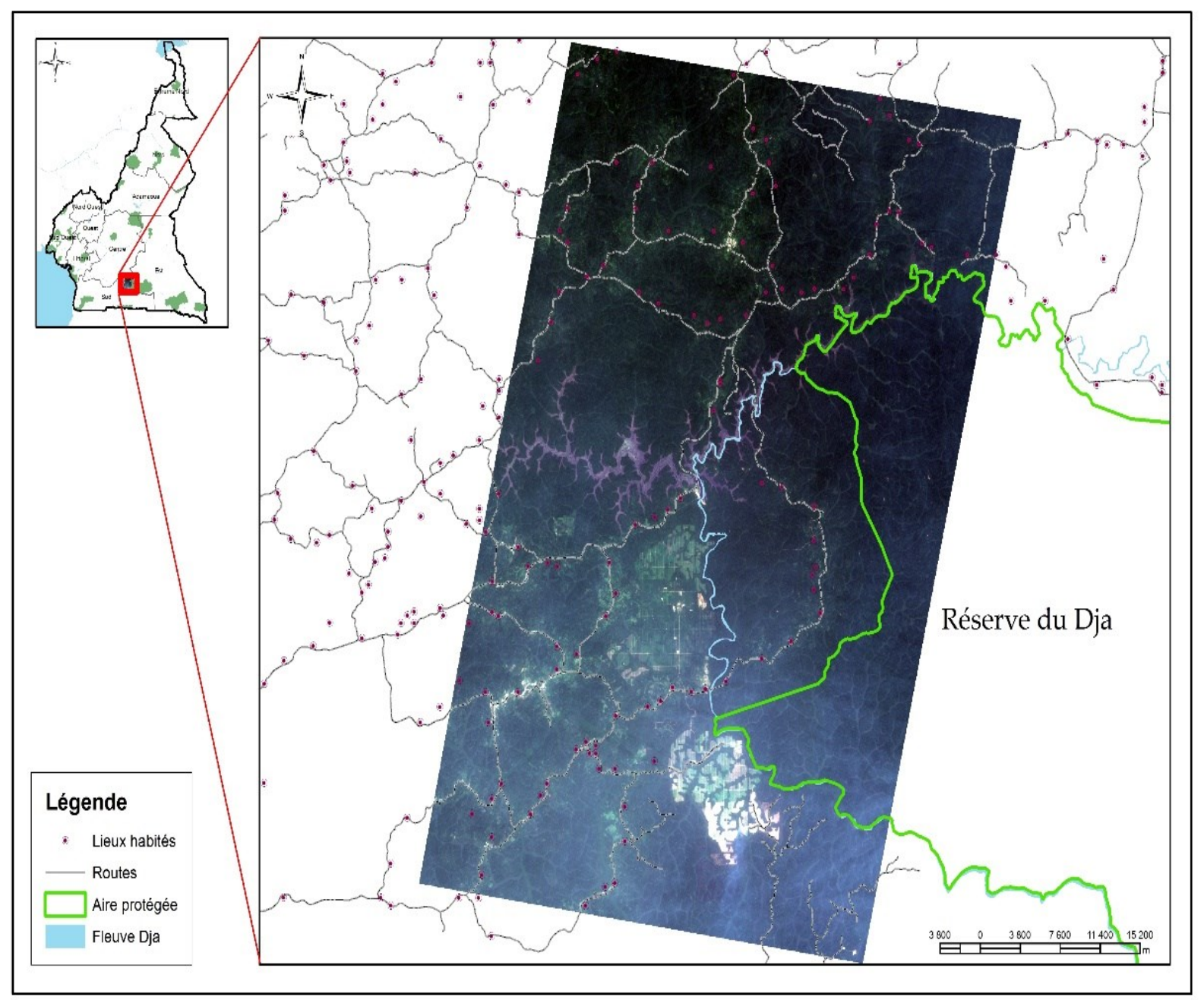

Figure 1 : Localisation de la zone d'étude

\subsection{Données utilisées}

Pour la réalisation de cette étude, huit images correspondant aux années 2011-2012-2014-2016 (ETM+), 2013-2015-2017-2018 (OLI), ont donc été téléchargées sur le site américain http://glovis.usgs.gov. Ces images ont été choisies sur le critère principal des saisons et de la couverture nuageuse (Andrieu and
Mering, 2008). Elles ont été choisies pour les mois de saison sèche afin de limiter le pourcentage de nuage et afin d'être pour le mieux possible dans la même saison végétale (Reis, 2008). Elles sont au nombre de huit pour permettre une visualisation année par année des changements. Les caractéristiques de ces images se trouvent dans le tableau 1. 


\begin{tabular}{|c|c|c|c|c|c|c|}
\hline Images & Satellites & Capteurs & Bandes & Couleurs & Longueur d'onde & Résolution \\
\hline \multirow{8}{*}{$\begin{array}{l}2011, \\
2012, \\
2014, \\
2016\end{array}$} & \multirow{8}{*}{ Landsat 7} & \multirow[t]{8}{*}{ ETM + } & Bande1 & Bleu & $0,45-0,52$ & \multirow[t]{5}{*}{$30 \times 30$} \\
\hline & & & Bande 2 & Vert & $0,52-0,60$ & \\
\hline & & & Bande 3 & Rouge & $0,63-0,69$ & \\
\hline & & & Bande 4 & PIR & $0,75-0,90$ & \\
\hline & & & Bande 5 & MIR & $1,55-1,75$ & \\
\hline & & & Bande 6 & TIR & $10,4-12,5$ & $60 \times 60$ \\
\hline & & & Bande 7 & MIR & $2,09-2,35$ & $30 \times 30$ \\
\hline & & & Bande 8 & PAN & $0,50-0,90$ & $15 \times 15$ \\
\hline \multirow{11}{*}{$\begin{array}{l}2013 \\
2015 \\
2017 \\
2018\end{array}$} & \multirow{11}{*}{ Landsat 8} & \multirow[t]{9}{*}{ OLI } & Bande 1 & Aérosols & $0,433-0,453$ & \multirow[t]{7}{*}{$30 \times 30$} \\
\hline & & & Bande 2 & Bleu & $0,45-0,515$ & \\
\hline & & & Bande 3 & Vert & $0,525-0,6$ & \\
\hline & & & Bande 4 & Rouge & $0,63-0,68$ & \\
\hline & & & Bande 5 & PIR & $0,845-0,885$ & \\
\hline & & & Bande 6 & MIR 1 & $1,56-1,66$ & \\
\hline & & & Bande 7 & MIR 2 & $2,1-2,3$ & \\
\hline & & & Bande 8 & PAN & $0,5-0,68$ & $15 \times 15$ \\
\hline & & & Bande 9 & CIRRUS & $1,360-1,390$ & $30 \times 30$ \\
\hline & & \multirow[t]{2}{*}{ TIRS } & $\begin{array}{l}\text { Bande } \\
10\end{array}$ & TIR & $10,3-11,3$ & \multirow[t]{2}{*}{$100 \times 100$} \\
\hline & & & $\begin{array}{l}\text { Bande } \\
11\end{array}$ & TIR & $11,5-12,5$ & \\
\hline
\end{tabular}

Tableau 1 : Caractéristiques des images Landsat utilisées (Wikipédia 2017)

\subsection{Méthode}

Les images une fois téléchargées ont été soumises à des prétraitements, notamment le co-géoréférencement, les corrections radiométriques (Rakotomala F.A and al 2015) et atmosphériques (Yirsaw et al. 2017), le layer stacking (Reis 2008b).

Dans un contexte d'étude de déforestation historique, le choix des classes d'occupation de sol s'est fait conformément aux orientations du Groupe d'experts intergouvernemental sur l'évolution du climat (IPCC 2008) décrivant les catégories d'activités qui contribuent aux émissions anthropiques de gaz à effet de serre. La définition des classes d'occupation de sol s'est également faite en fonction de l'interprétation spectrale (compositions colorées et analyse de profils spectraux) des images satellites (Bickel et al. 2006) et sur l'utilisation d'images de hautes résolution (Google Earth). Le tableau 2 donne des informations sur les classes d'occupation de sol définies.

\begin{tabular}{|c|l|l|}
\hline $\mathbf{N}^{\circ}$ & \multicolumn{1}{|c|}{ Classes } & \multicolumn{1}{c|}{ Caractéristiques } \\
\hline 1 & Forêt dense & $\begin{array}{l}\text { Cette catégorie comprend toutes les terres avec une végétation dense et } \\
\text { ligneuse. }\end{array}$ \\
\hline 2 & Forêt dégradée & Surface avec peu de végétation ligneuse et faiblement dense. \\
\hline 3 & Zone de culture & $\begin{array}{l}\text { Cette catégorie comprend les terres cultivées et les systèmes d'agroforesterie. la } \\
\text { réponse spectrale est plus élevée que pour les forêts car ici la végétation est } \\
\text { jeune et saine }\end{array}$ \\
\hline 4 & Zone marécageuse & $\begin{array}{l}\text { Cette catégorie comprend les zones et les terres recouvertes ou saturées d'eau } \\
\text { pendant tout ou partie de l'année }\end{array}$ \\
\hline 5 & Zone d'habitation & $\begin{array}{l}\text { Cette catégorie comprend toutes les terres artificialisées, y compris les } \\
\text { infrastructures de transport et les établissements humains de toute taille }\end{array}$ \\
\hline 6 & Sol nu & $\begin{array}{l}\text { Cette catégorie est caractérisée par l'absence de végétation et est souvent } \\
\text { confondue avec les zones habitées, en raison de la similitude des réponses } \\
\text { spectrales. }\end{array}$ \\
\hline 7 & Eau & \begin{tabular}{l} 
Sont inclus dans cette catégorie, les rivières, ruisseaux et lacs \\
\hline
\end{tabular}
\end{tabular}

Tableau 2 : Classes d'occupation de sol identifiées 
Pour extraire les types d'occupation du sol sur les images traitées, la technique de classification supervisée par maximum de vraisemblance (MLC) a été utilisée grâce à des signatures collectées parmi les 7 classes identifiées (Rahman, Aldosary, and Mortoja 2017). Le logiciel Erdas Imagine 2014 muni de l'algorithme de classification supervisée par maximum de vraisemblance a été utilisé pour réaliser les différentes cartes d'occupation des sols. Pour vérifier la qualité de l'exercice de classification, une évaluation a été faite (Wachiye 2013). La validation des classifications a été réalisée à l'aide d'un outil statistique inclus dans le logiciel Erdas Imagine 2014. Cette validation était basée sur la comparaison des résultats de la classification avec une imagerie de référence (images d'archive Google Earth) (BAYSAL 2013).

La méthode utilisée pour ressortir les points chauds de déforestation est l'analyse par "change détection " (Usha, Anitha, and lyappan 2012), entre les images résultant de la classification après harmonisation de celles-ci (Mishra, Rai, and Mohan 2014). Cette technique a été appliquée grâce au module « change analysis " du logiciel Idrisi/Terrset. L'analyse des changements s'est faite à partir des images d'occupation du sol (2011 et 2018). Les changements identifiés représentent des transitions d'un type d'occupation du sol à un autre (Eastman 2016). La figure 2 résume les étapes de la méthodologie générale de cette étude.

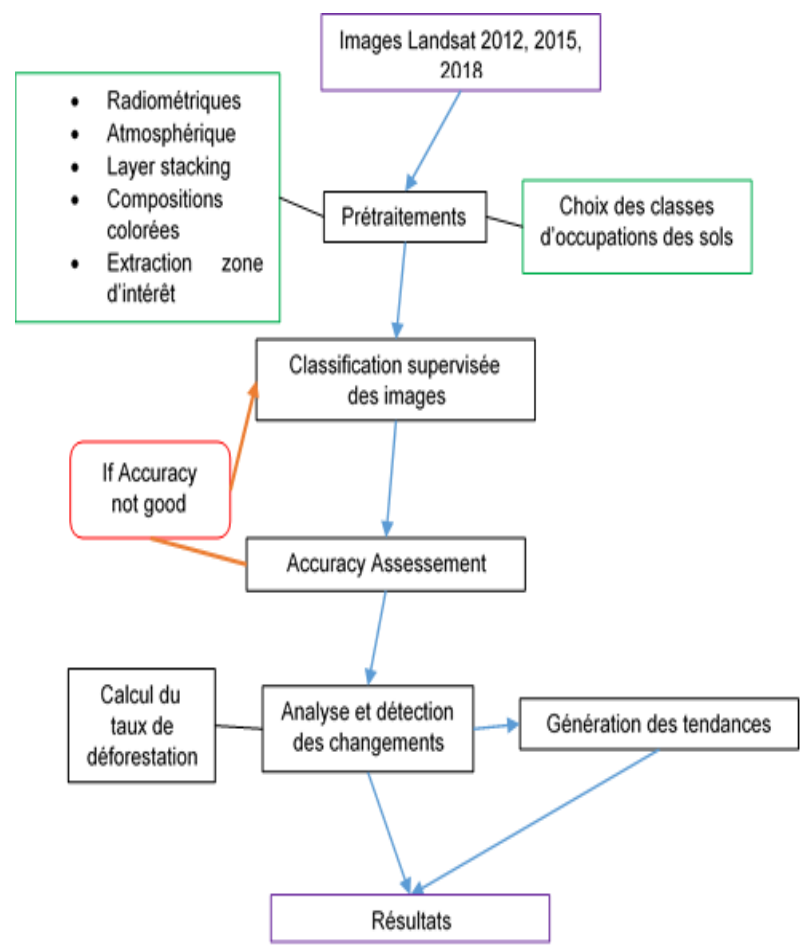

Figure 2 : Diagramme méthodologique de l'étude
Le taux de déforestation a été également calculé pour ressortir l'ampleur de la déforestation. Plusieurs publications ont récemment mis en lumière que l'évolution de la déforestation ne peut pas être obtenue avec ce simple rapport (Puyravaud 2002). La formule standardisée proposée par Puyravaud, (2002) a été utilisée pour calculer le taux de déforestation cf. équation (1).

$$
\mathrm{T}=-\frac{1}{\mathrm{t} 2-\mathrm{t} 1} \ln \left(\frac{\mathrm{A} 2}{\mathrm{~A} 1}\right) * 100
$$

Avec :

A1 = Superficie de la classe forestière pour l'année initiale

A2 = Superficie de la classe forestière pour l'année finale

$\mathrm{T}=$ Taux de déforestation

$\mathrm{t} 1$ = Date de l'année initiale

t2 = Date de l'année finale

\section{Résultats}

\subsection{Classification de l'occupation du} sol sur les trois années

La qualité des classifications et des cartes obtenues peut être considérée comme bonne, au regard du degré d'accord du coefficient Kappa présenté dans le tableau 3. Les coefficients de Kappa obtenus après les différentes classifications (Tableau 4), se retrouvent dans l'intervalle [0.80 -0.61 , ce qui permet de conclure que les classifications ont été bien menées.

\begin{tabular}{|l|l|}
\hline Accord & Kappa \\
\hline Excellent & 0,81 \\
\hline Bon & $0,80-0,61$ \\
\hline Modéré & $0,60-0,41$ \\
\hline Médiocre & $0,40-0,21$ \\
\hline Mauvais & $0,20-0,0$ \\
\hline Très mauvais & $<0,0$ \\
\hline
\end{tabular}

Tableau 3 : Statistiques globales de Kappa (Landis and Koch 1977)

\begin{tabular}{|l|l|l|l|}
\hline Classifications & 2012 & 2015 & 2018 \\
\hline Kappa & 0.64 & 0.67 & 0.76 \\
\hline
\end{tabular}

Tableau 4 : Coefficients de Kappa pour chaque classification

La figure 3 présente les différentes cartes d'occupation du sol pour les années choisies tandis que la figure 4 montre l'évolution des classes d'occupation du sol pendant la période 2011 à 2018.

La figure 5 est la carte des changements apparus dans la zone pendant cette même période. Le tableau 5 quant à lui montre les superficies de chaque classe pour les différentes années. 


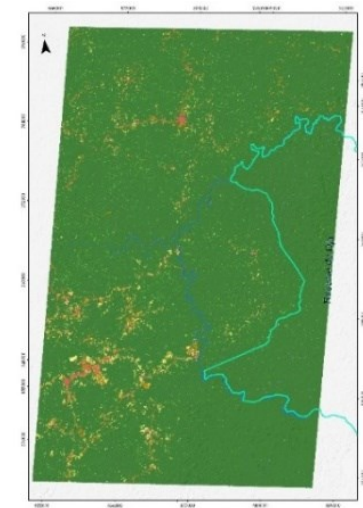

2011

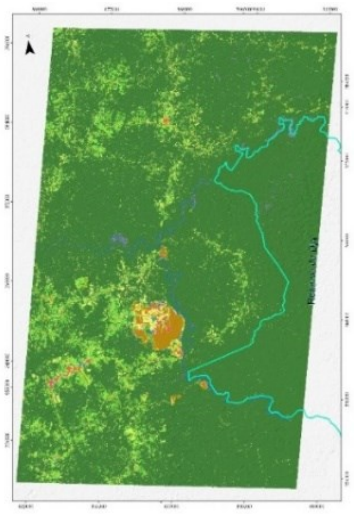

2015

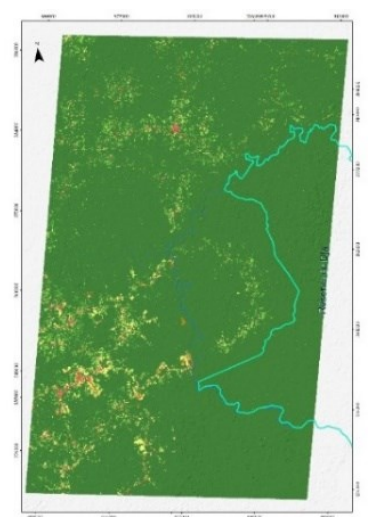

2012

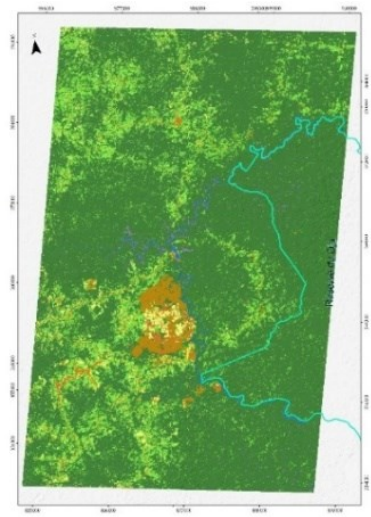

2016

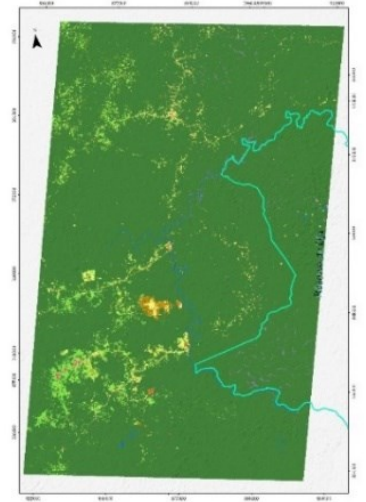

2013

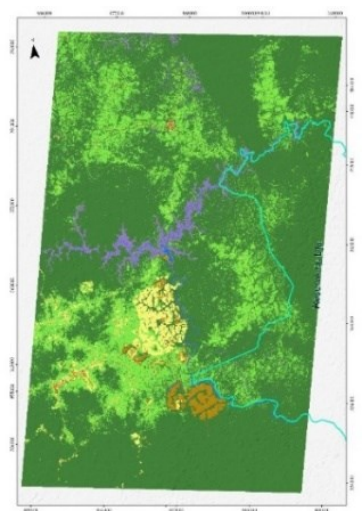

2017

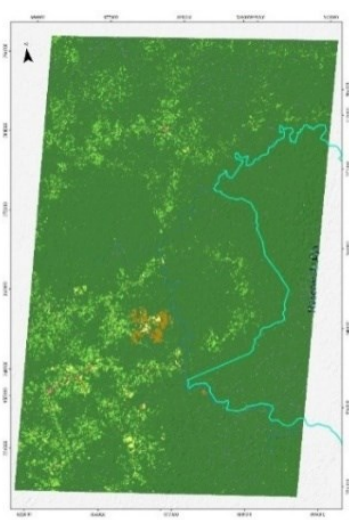

2014

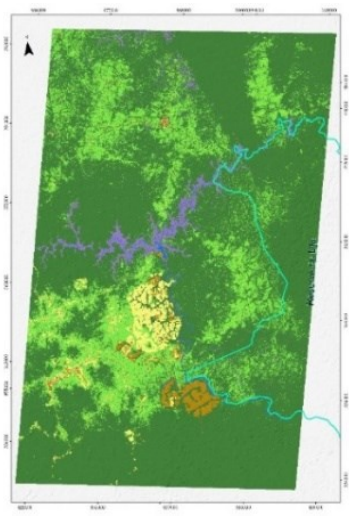

2018

\section{Légende}

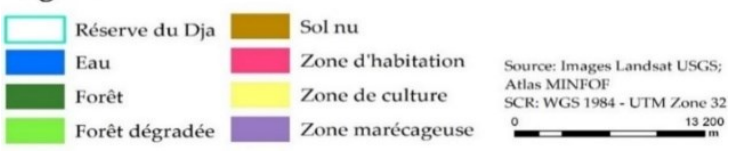

Figure 3 : Cartes d'occupation du sol

\begin{tabular}{|l|r|r|r|r|r|r|r|r|}
\multicolumn{10}{c|}{ Superficies en Ha } \\
\hline Classes & $\mathbf{2 0 1 1}$ & $\mathbf{2 0 1 2}$ & $\mathbf{2 0 1 3}$ & $\mathbf{2 0 1 4}$ & $\mathbf{2 0 1 5}$ & $\mathbf{2 0 1 6}$ & $\mathbf{2 0 1 7}$ & $\mathbf{2 0 1 8}$ \\
\hline Forêt dégradée & 4624.83 & 8686.44 & 8204.49 & 17580.8 & 21186.7 & 47640.5 & 57865 & 72901.2 \\
\hline Zone de culture & 5231.61 & 5909.85 & 5383.08 & 2181.6 & 13213.3 & 8286.57 & 8784.09 & 12294.1 \\
\hline Zone d'habitation & 195.21 & 301.14 & 710.28 & 722.79 & 774.9 & 1010.88 & 1365.12 & 1568.79 \\
\hline Zone marécageuse & 2555.91 & 597.6 & 1977.48 & 5273.64 & 2675.97 & 3275.1 & 8805.96 & 10895.9 \\
\hline Sol nu & 4979.97 & 1368.18 & 2168.55 & 1233.9 & 4939.74 & 10361.5 & 4215.06 & 7830.72 \\
\hline Eau & 753.39 & 886.32 & 1125.81 & 1178.37 & 1196.19 & 1401.94 & 1608.94 & 1494.45 \\
\hline Forêt dense & 279954 & 280557.8 & 278737.6 & 270136.4 & 254320.8 & 226273 & 215663.3 & 191322.4 \\
\hline
\end{tabular}

Tableau 5 : Résultats statistiques des cartes d'occupation du sol

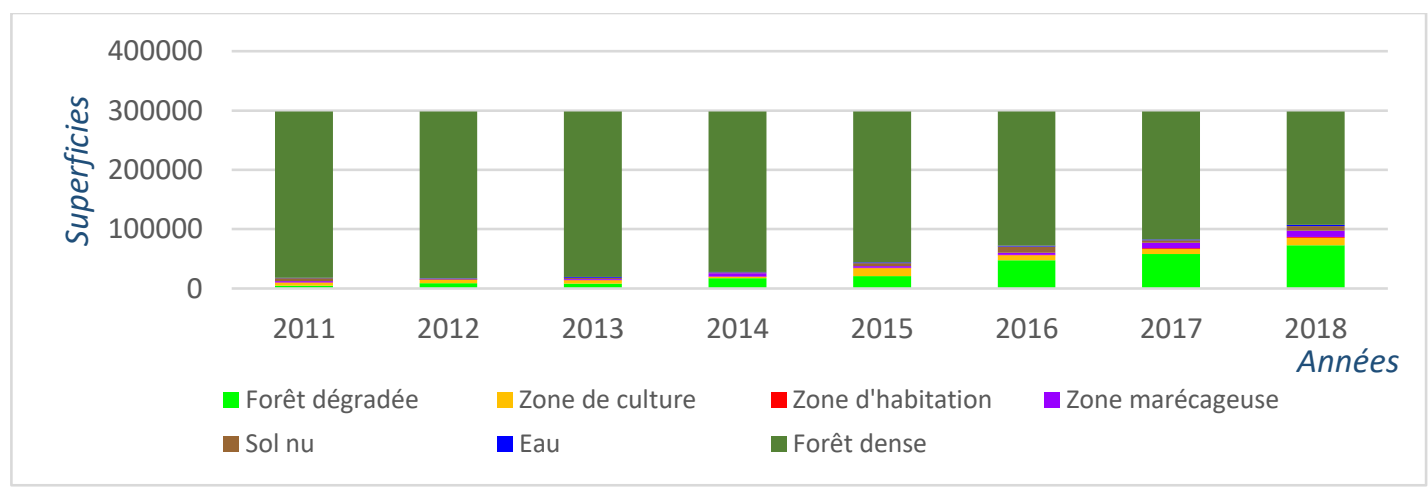

Figure 4 : Evolution de l'occupation du sol entre 2011 et 2018 


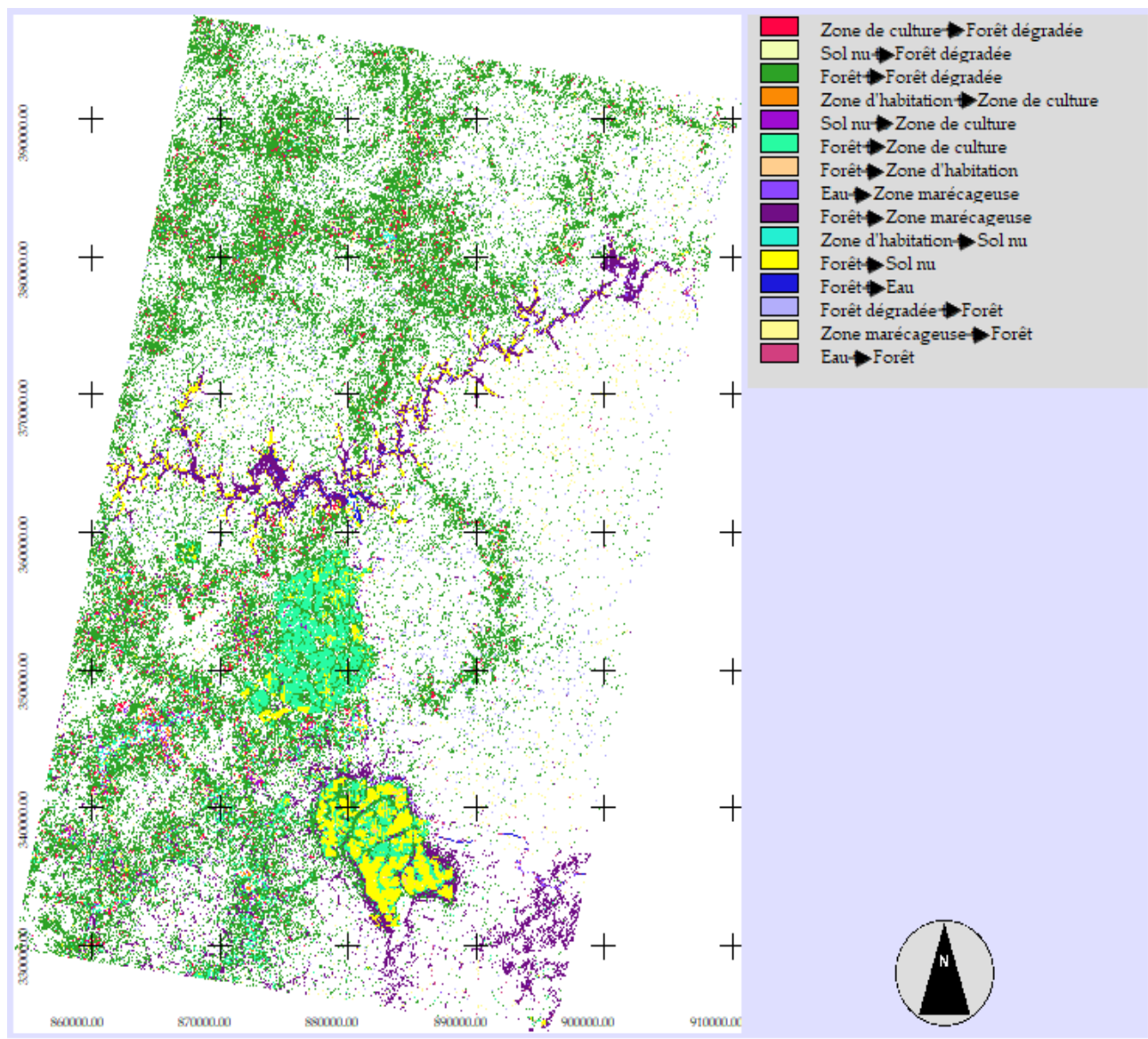

Figure 5 : Carte des changements

Ces résultats montrent une importante dynamique de l'occupation du sol pendant la période 2012 - 2018 . C'est le cas particulièrement de la végétation (forêt, forêt dégradée), des zones de culture, des marécages et des zones d'habitation.

\begin{tabular}{|l|r|r|r|r|}
\hline & $\mathbf{2 0 1 1}$ & $\mathbf{2 0 1 8}$ & $\begin{array}{l}\text { 2018- } \\
\text { 2011 }\end{array}$ & $\begin{array}{l}\text { (2018- } \\
\mathbf{2 0 1 1})\end{array}$ \\
\hline Forêt dégradée & 4624,83 & 72901,2 & 68276,37 & 22,88 \\
\hline Zone de culture & 5231,61 & 12294,1 & 7062,49 & 2,36 \\
\hline Zone d'habitation & 195,21 & 1568,79 & 1373,58 & 0,46 \\
\hline Zone marécageuse & 2555,91 & 10895,9 & 8339,99 & 2,79 \\
\hline Sol nu & 4979,97 & 7830,72 & 2850,75 & 0,95 \\
\hline Eau & 753,39 & 1494,45 & 741,06 & 0,24 \\
\hline Forêt dense & 279954 & 191322,4 & $-88631,6$ & $-29,71$ \\
\hline
\end{tabular}

Tableau 6 : Pourcentage de changement d'occupation du sol

\subsection{Cartographie de la déforestation}

Le résultat des classifications montre que les zones forestières denses ont diminué, alors que toutes les autres classes ont augmenté. Une grande partie de ces forêts denses se sont transformées en forêts dégradées, en zones de culture, en zone marécageuses, et en sol nu comme l'indiquent les pourcentages de changement dans le tableau 6. La figure 6 montre la contribution de ces principaux types d'occupation du sol dans les changements survenus au niveau des surfaces forestières denses. On observe qu'effectivement, l'extension des zones de culture, les sols nus, les zones marécageuses et les forêts dégradées ont contribué significativement à la baisse du couvert forestier dense.

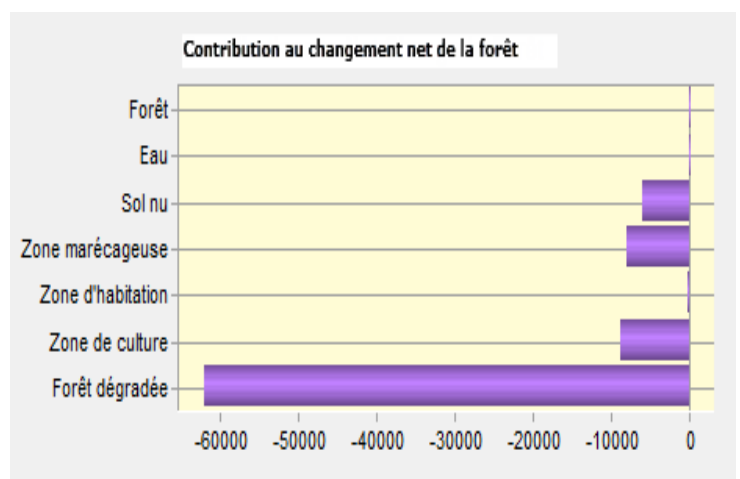

Figure 6 : Principaux contributeurs aux changements du couvert forestier

Le taux de déforestation a été évalué à $6,8 \%$. Ce taux indique la pression importante exercée sur les ressources forestières dans cette périphérie de la réserve. La figure 7 présente les zones de déforestation apparues pendant cette période d'étude. Les figures 8 , 9, 10, 11 montrent les transitions nettes des surfaces forestières denses vers les contributeurs majeurs identifiés. 


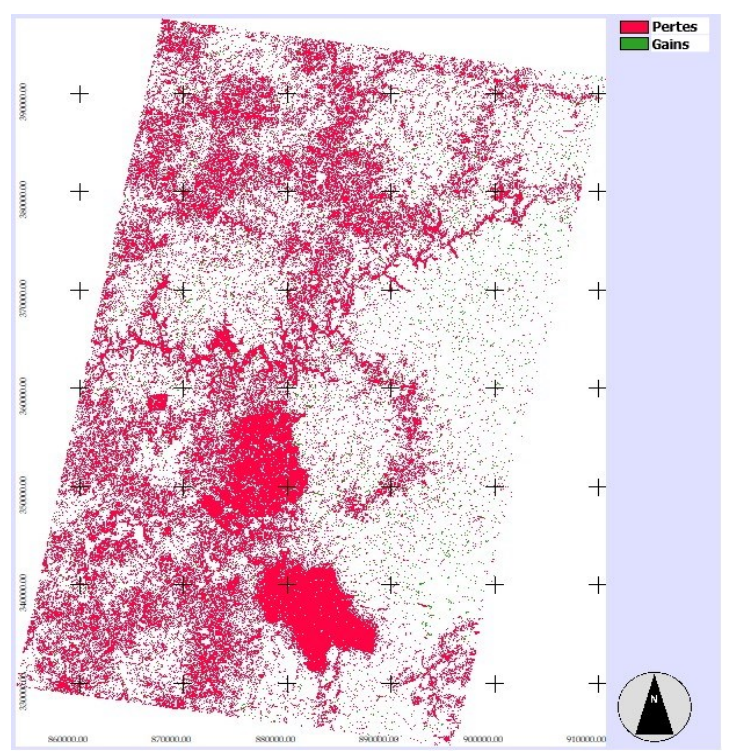

Figure 7 : Déforestation observée entre 2012 et 2018

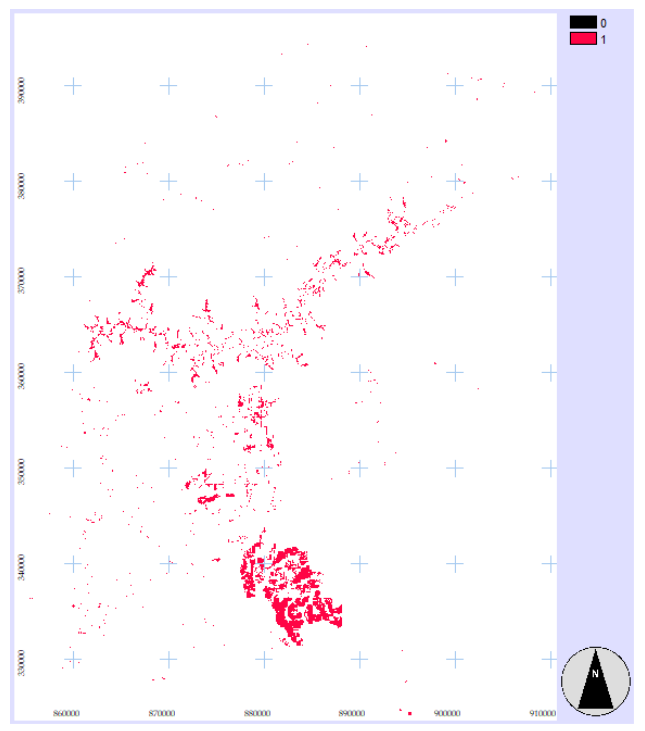

Figure 9 : Forêt dense à sol nu

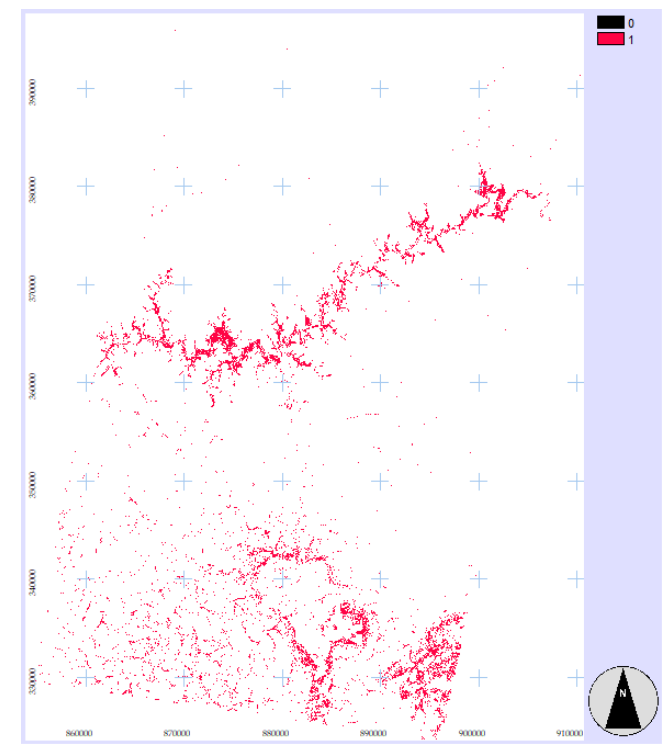

Figure 11 : Forêt dense à zone marécageuse



Figure 8 : Forêt dense à forêt dégradée

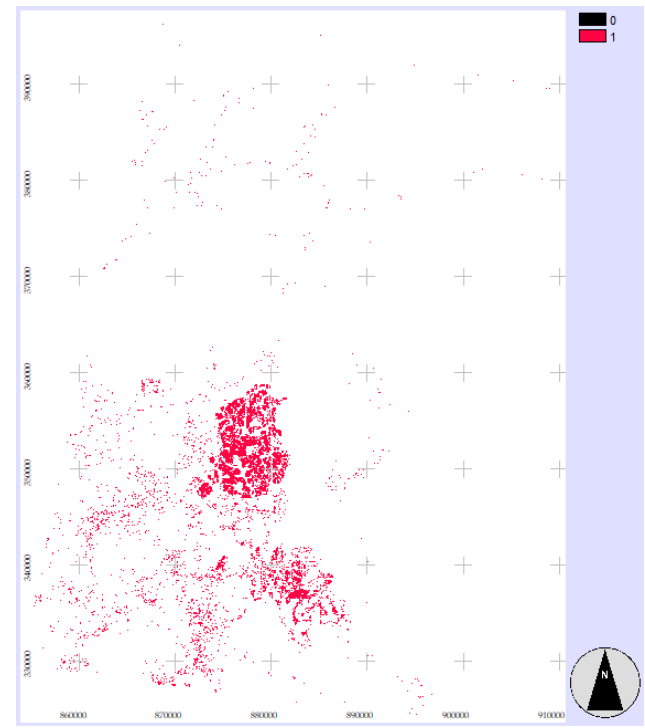

Figure 10 : Forêt dense à zone de culture 


\subsection{Evaluation des tendances spatiales de déforestation en fonction des contributeurs majeurs}

Cette section a consisté en la création de cartes montrant les zones ayant des tendances fortes pour

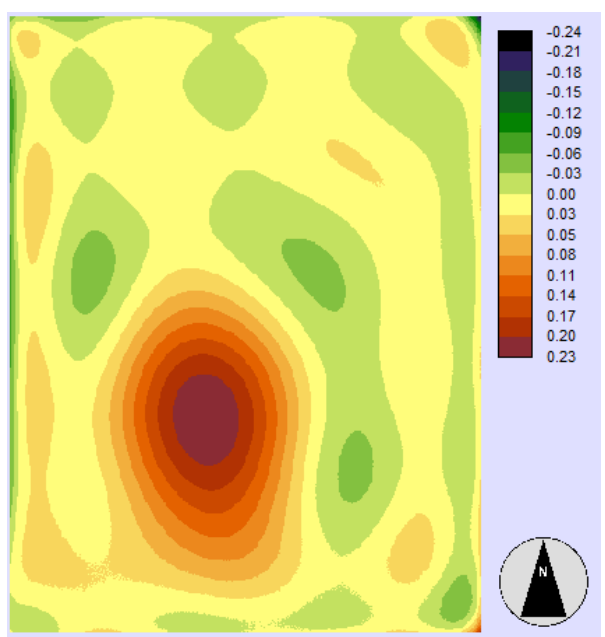

Figure 12 : Tendance de déforestation par rapport aux zones de culture

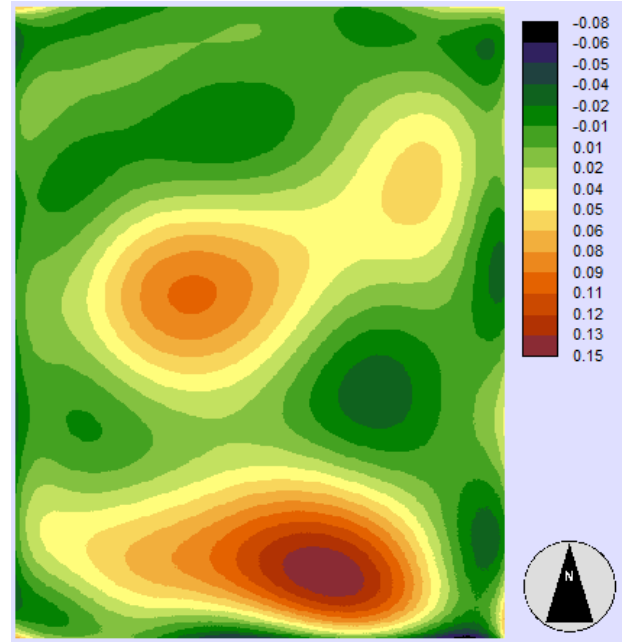

Figure 14 : Tendance de déforestation par rapport aux zones marécageuses

\section{Discussion}

La périphérie ouest de la réserve du Dja est une zone sensible à l'avancée de la déforestation. La dynamique importante de l'occupation du sol qui a eu lieu pendant ces sept dernières années se caractérise par un taux élevé de déforestation. Cette étude permet de constater que la perte du couvert forestier dense a été importante entre 2011 et 2018

Cette étude permet aussi de montrer que les surfaces forestières denses se sont principalement converties en forêt dégradées, en sols nus, en zones de culture et en zones marécageuses. Entre 2011 et 2014, la la perte du couvert forestier dense dans la zone d'étude en fonction des contributeurs principaux identifiés. Les figures $12,13,14,15$ présentent les zones de tendance à la déforestation. Au niveau de la légende, elles correspondent aux zones ayant les valeurs positives les plus élevées.

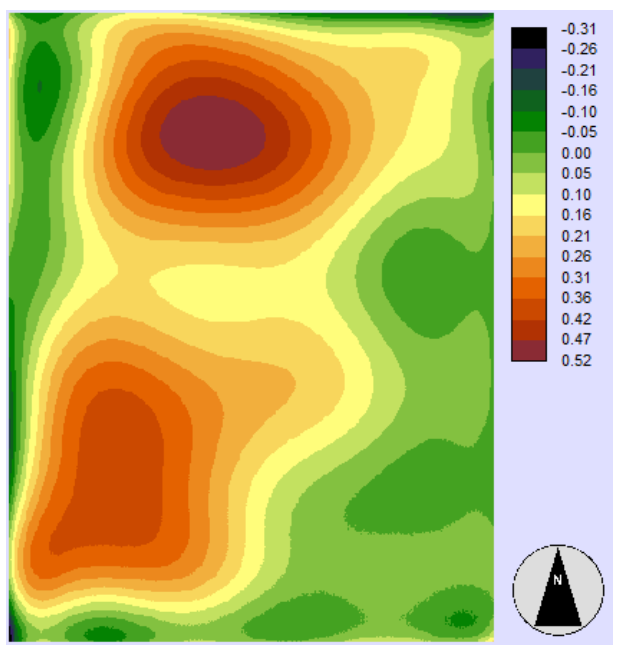

Figure 13 : Tendance de déforestation par rapport aux forêts dégradées

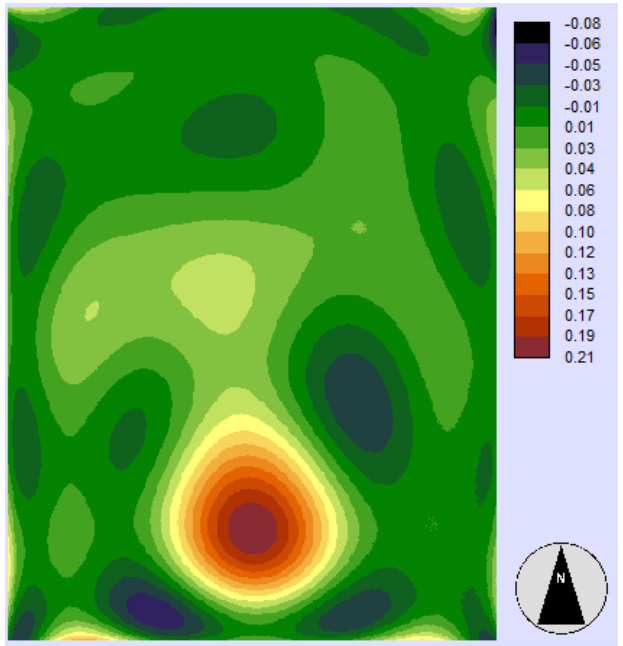

Figure 15 : Tendance de déforestation par rapport aux sols nus

déforestation était très faible. Elle est devenue forte à partir de 2015. En effet sur le plan des activités anthropiques, c'est pratiquement en 2015 que débute la mise en place de la plantation industrielle d'hévéa (SUDCAM) et l'enlèvement de la biomasse forestière dans la retenue du barrage de Mekin. Ces deux faits combinés aux pratiques paysannes de l'agriculture, à la densité de la population et aux infrastructures (réseau routier), sont responsables en grande partie de la baisse rapide du couvert forestier dense. La figure 16 montre la corrélation visuelle qui existe entre la déforestation apparue et ces moteurs. 


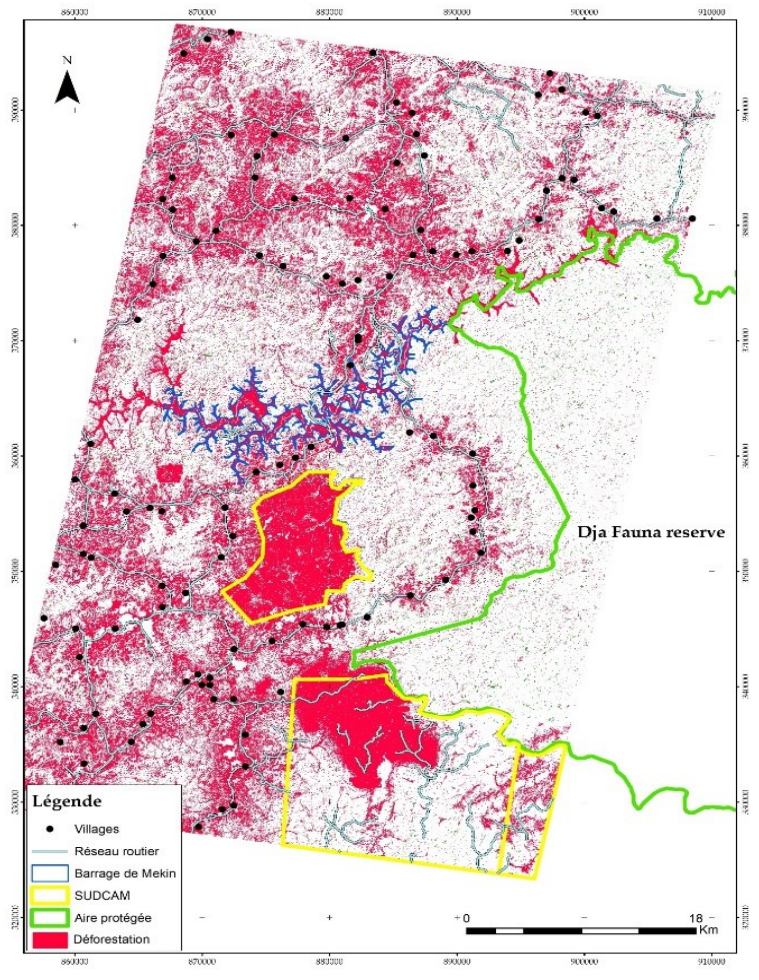

Figure 16 : Corrélation entre la déforestation et les moteurs

On observe que la répartition des surfaces forestières dégradées est à peu près semblable à celle des zones d'habitation. On peut donc dire que la densité de la population avec les activités de coupe du bois et des feux de brousse est responsable de la formation des forêts dégradées. La conversion en sol nu n'est qu'une transition vers les zones de culture. Ce fait s'observe avec acuité au niveau de la plantation industrielle SUDCAM. Pour la conversion en zone marécageuse, même si elle est répartie dans la partie sud de la zone d'étude, elle est néanmoins concentrée au niveau de la retenue du barrage de Mekin (Figure 10).

Malheureusement, plusieurs autres activités anthropiques sont planifiées dans cette périphérie ouest de la RBD par le gouvernement. C'est le cas de la perspective d'un projet d'exploitation minière attribué aux concessionnaires Venture Capital PLC et Zamba Holding et de l'extension du réseau électrique dans cette zone. Combinées aux autres facteurs identifiés comme contributeurs, on peut entrevoir une pression encore plus grande sur les ressources forestières. Le taux de déforestation déjà élevé pour ces 7 dernières années pourrait augmenter considérablement. Des perspectives pour cette étude pourraient être de continuer le suivi annuel par télédétection, en appliquant la même méthode année par année, mais aussi de considérer la déforestation actuelle $(\mathrm{t})$ comme ligne de base et de faire une modélisation future $(t+7)$ de cette déforestation. Cette modélisation pourra permettre de délimiter une zone de référence dédiée à la conservation des forêts et donc des stocks de carbone dans l'optique de la mise en œuvre d'un projet REDD+.

\section{Conclusion}

Le but de cette étude était de cartographier la déforestation dans la périphérie ouest de la réserve de biosphère du Dja. Pour y parvenir, la technique de télédétection à travers les analyses de classification supervisée et de " change detection " a été utilisée sur la période 2011 - 2018. Les résultats de cette étude montrent que la déforestation est une problématique importante dans la périphérie de la réserve. En effet le taux annuel de déforestation observé est élevé $(5,4 \%)$. Cela est révélé par la forte baisse du couvert forestier dense (-29\%). Au cours de ces 7 années les surfaces forestières denses se sont principalement transformées en forêt dégradée, sol nu, zone marécageuse et zone de culture. Ces contributeurs à la déforestation correspondent aux activités anthropiques qui ont lieu dans la zone. La localisation spatiale de ces contributeurs correspond aux zones tendancieuses à la déforestation qui ont été identifiées. Les contributeurs à cette déforestation pourront être utilisés dans une autre étude pour modéliser la déforestation future à l'horizon 2035, dans l'optique de parvenir à la délimitation d'une zone de référence qui sera destinée à la conservation des forêts denses.

\section{Remerciements}

Même si la réalisation de cette étude s'est faite avec nos propres moyens, nous souhaitons tout de même remercier le responsable du Centre de Recherche spécialisé sur Forêt et Environnement pour son accompagnement et sa participation. Nous remercions également nos collègues chercheurs pour les idées et critiques qu'ils ont formulées pour l'amélioration de ce travail. Enfin, nous remercions les chefs et les habitants des villages visités pour leur disponibilité pendant la mission de terrain.

\section{Références}

Andrieu, Julien, and Catherine Mering 2008. Cartographie Par Télédétection Des Changements de La Couverture Végétale Sur La Bande Littorale OuestAfricaine : Exemple Des Rivières Du Sud Du Delta Du Saloum Au Rio Geba: 27.

Baysal, Gûlendam 2013. Urban Land Use and Land Cover Change analysis and Modeling A case study area Malatya, Turkey: 75.

Bickel, Kathryn, Richard Gary, Michael Köh, and Vianna Rodrigue 2006. Consistent representation of Lands. Agriculture, Forestry and Other Land Use Volume 4. 2006 IPCC Guidelines for National Greenhouse Gas Inventories.

CVUC 2014 MEYOMESSALA.

http://cvuc.cm/national/index.php/fr/carte-

communale/region-du-sud/90-association/carteadministrative/314-meyomessala, accessed March 22, 2018

Eastman, J. Ronald 2016 TerrSet Tutorial, Geospatial Monitoring and Modeling System. Clark Labs.

Engono M, Adrienne $2017 \quad$ La Réserve Du Dja Risque de Perdre Son Statut de Patrimoine Mondial de I'Unesco |. http://infocongo.org/la-reserve-du-dja-risquede-perdre-son-statut-de-patrimoine-mondial

delunesco/?lang=fr $\# ! / m a p=209 \& l o c=3.1075186500000$ 04,13.014640165688984,12, accessed June 18, 2018. 
Greenpeace 2016 L'UNESCO Échoue à Protéger La Réserve Du Dja Au Cameroun de Multiples Menaces, y Compris La Plantation d'hévéa Sudcam. Greenpeace Afrique. accessed http://www.greenpeace.org/africa/fr/Actualities/actualite s/LUNESCO-echoue-a-proteger-la-Reserve-du-Dja-auCameroun-de-multiples-menaces-y-compris-laplantation-dhevea-Sudcam/, September 18, 2017.

IPCC 20082006 IPCC Guidelines for National Greenhouse Gas Inventories - A Primer, Prepared by the National Greenhouse Gas Inventories Programme, Eggleston H.S., Miwa K., Srivastava N. and Tanabe K. (Eds). IGES, Japan : 12.

Mansourian, S., A. Belokurov, and P.J. Stephenson 2017 Rôle Des Aires Protégées Forestières Dans l'adaptation Aux Changements Climatiques. Archives de Documents de La FAO. accessed http://www.fao.org/docrep/011/i0670f/i0670f13.htm, June 28, 2017.

MINFOF, and UICN 2015 Caractérisation d'une population de grands et moyens mammifères dans la Reserve de Faune du Dja: Potentiel et menaces. Yaoundé.

Mishra, Varun, Praveen Rai, and Kshitij Mohan 2014. Prediction of Land Use Changes Based on Land Change Modeler (LCM) Using Remote Sensing: A Case Study of Muzaffarpur (Bihar), India. Journal of the Geographical Institute Jovan Cvijic, SASA 64(1): 111-127.

N'Da, Hippolyte Dibi, Edouard Kouakou N'Guessan, Mathieu Egnankou Wajda, and Kouadio Affian 2008. Apport de la télédétection au suivi de la déforestation dans le Parc National de la Marahoué (Côte d'Ivoire). Revue Télédétection 8(1) : 17-34.

Ngamba, Mikwa, Masimo Kabuang'a, Anitambua Sungu, Jean-Marie Kahindo, and Hyppolite Nshimba 2016. Analyse prospective de la déforestation estimée par télédétection dans la réserve de biosphère de Yangambi 24(2) : 19.

Niang, Moustapha, Olivier Hamerlynck, and Cédric Hance 2006. Rapport de Mission Suivi de l'état de La Conservation de La Réserve de Faune de Dja En République Du Cameroun, Site de Patrimoine Mondial. UNESCO \& IUCN, Paris \& Switzerland.http://whc.unesco.org/archive/2007/mis407jun2006.pdf, accessed June 28, 2017.

Oke, Ndum Fidelis 2009 Deforestation Impacts on Biodiversity Conservation in the Dja Biosphere Reserve of Cameroon. http://www.divaportal.org/smash/record.jsf?pid=diva2:346164, accessed June 28, 2017.
PNDP 2016 Plan Communal de développement de Meyomessala. PNDP.

Puyravaud, J. P. 2002 Standardizing the Calculation of the Annual Rate of Deforestation. Ecology and Management: 177: pp 593-596.

Rahman, Muhammad Tauhidur, Adel S. Aldosary, and Md. Golam Mortoja 2017. Modeling Future Land Cover Changes and Their Effects on the Land Surface Temperatures in the Saudi Arabian Eastern Coastal City of Dammam. Land 6(4): 36.

Rakotomala F.A, and al 2015 Estimation de la déforestation des forêts humides à Madagascar utilisant une classification multidate d'images Landsat entre 2005, 2010 et 2013. Revue Française de Photogrammétrie et de Télédétection.

Rautner, Leggett, M., and Davis, F 2013 Le Petit Livre Des Grands Moteurs de Déforestation. Global Canopy Programme: Oxford.

Reis, Selçuk 2008a. Analyzing Land Use/Land Cover Changes Using Remote Sensing and GIS in Rize, NorthEast Turkey. Sensors 8(10): 6188-6202.

2008b Analyzing Land Use/Land Cover Changes Using Remote Sensing and GIS in Rize, North-East Turkey. Sensors 8(10): 6188-6202.

Solefack, Marie Caroline Momo, Olivier Chabrerie, Emilie Gallet-Moron, et al. 2012 Analyse de la dynamique de déforestation par télédétection couplée aux modèles d'équations structurales : exemple de la forêt néphéliphile du mont Oku (Cameroun). Acta Botanica Gallica 159(4): 451-466.

Usha, M., K. Anitha, and L. Iyappan 2012. Landuse Change Detection through Image Processing and Remote Sensing Approach: A Case Study of Palladam Taluk, Tamil Nadu. International Journal of Engineering Research and Applications 2(4): 289-294.

Verjans, par Vincent 2016. La Déforestation Aujourd'hui. Le Journal International. http://www.lejournalinternational.info/la-deforestationaujourdhui/, accessed June 29, 2017.

Wachiye, Aswani 2013GIS Based Forest Cover Change and Vulnerability Analysis: A Case Study of the Nandi North Forest Zone. Journal of Geography and Regional Planning 6(5): 159-171.

Yirsaw, Eshetu, Wei Wu, Xiaoping Shi, Habtamu Temesgen, and Belew Bekele 2017 Land Use/Land Cover Change Modeling and the Prediction of Subsequent Changes in Ecosystem Service Values in a Coastal Area of China, the Su-Xi-Chang Region. Sustainability 9(12): 120 . 